Pacific Journal of Mathematics

EXCEPTIONAL SETS FOR POISSON INTEGRALS OF
POTENTIALS ON THE UNIT SPHERE IN $C^{n}, p \leq 1$ 


\title{
EXCEPTIONAL SETS FOR POISSON INTEGRALS OF POTENTIALS ON THE UNIT SPHERE IN $\mathbf{C}^{n}, p \leq 1$
}

\section{Patrick Ahern and Carmen Cascante}

\begin{abstract}
In this article we show that the exceptional sets for Poisson-Szegö integrals of potentials of $H^{p}$ functions in the unit ball in $\mathrm{C}^{n}$ have a certain Hausdorff measure zero, and that this result is sharp.
\end{abstract}

Let $B^{n}$ denote the unit ball in $\mathbf{C}^{n}$ with boundary $S, \sigma$ will denote the normalized Lebesgue measure on $S$. We let $R$ denote the (holomorphic) radial derivative $R=\sum_{j=1}^{n} z_{j} \partial / \partial z_{j}$. A holomorphic function $f$ belongs to $\mathscr{H}^{p}$ if $\sup _{0<r<1} \int_{s}|f(r \zeta)|^{p} d \sigma(\zeta)<\infty$. In [2] and [5] it was shown that if $R^{k} f \in \mathscr{H}^{p}$ where $0<p \leq 1$ and $n-k p>0$ then the function $f$ has an admissible limit on $S \backslash E$ where $E$ has non-isotropic Hausdorff measure zero in dimension $m=n-k p$, and this result is sharp. For $p>1$, the proper measure for the exceptional sets is a certain capacity; see [4]. In [1] D. Adams proved an analogous result for harmonic functions, see also [2]. For harmonic functions the result is the following: if $u$ is a fractional integral of order $\beta$ (i.e. Bessel potential) of an $H^{p}\left(R^{n}\right)$ distribution, $0<p \leq 1$, then the Poisson integral of $u$ has non-tangential limits on $R^{n} \backslash E$ where $E$ has Hausdorff measure zero in dimension $m=n-\beta p$. Again, for $p>1$, the proper measure of the exceptional sets is capacity.

In this paper we prove an analogous result for certain non-isotropic potentials on $S$. If $k$ is a positive integer, $k<n$, we let

$$
I_{k}(z, \zeta)=|1-\langle z, \zeta\rangle|^{k-n}, \quad z, \zeta \in S .
$$

For a function $v$ on $S$ let

$$
\left(I_{k} v\right)(z)=\int_{S} I_{k}(z, \zeta) v(\zeta) d \sigma(\zeta)
$$

The kernels $I_{k}$ will play the role of the Bessel kernels in $R^{n}$. Indeed, $I_{1}$ is the fundamental solution for a certain sublaplacian on $S$, see [9]. In contrast to the cases mentioned above we can handle only the case where $k$ is an integer. If

$$
P(z, \zeta)=\frac{\left(1-|z|^{2}\right)^{n}}{|1-\langle z, \zeta\rangle|^{2 n}}, \quad z \in B^{n}, \zeta \in S,
$$


is the Poisson-Szegő kernel we are interested in exceptional sets of functions

$$
P\left[I_{k} v\right](z)=\int_{S} P(z, \zeta)\left(I_{k} v\right)(\zeta) d \sigma(\zeta)
$$

where $v$ is a distribution in the atomic Hardy space $H^{p}(S), 0<p \leq$ 1 , of Garnett and Latter [7]. We will show that the set where such a function fails to have an admissible limit has non-isotropic Hausdorff measure zero in dimension $m=n-k p$. The method of [2] shows the following: if $u$ is a continuous function in $B^{n}$ whose admissible maximal function $M u \in L^{p}(d \sigma), 0<p \leq 1$, and if

$$
F(z)=\int_{0}^{1}\left(\log \frac{1}{t}\right)^{k-1} u(t z) d t
$$

where $n-k p>0$ then the admissible maximal function $M F \in$ $L^{p}(d \nu)$ for any measure $\nu$ on $S$ that satisfies $\nu(B(\zeta, \delta)) \leq \delta^{n-k p}$ for all $B(\zeta, \delta)=\{y \in S:|1-\langle\zeta, n\rangle|<\delta\}$. If we knew this to be true for all $F=P\left[I_{k} v\right], v \in H^{p}$, then it would follow in a standard way that all such $P\left[I_{k} v\right]$ have admissible limits on the complement of a set whose non-isotropic Hausdorff measure is zero in dimension $n-k p$, see [2] and [5]. Assuming this, our problem reduces to the following: Given $v \in H^{p}, 0<p \leq 1$, show that there is a $u$ with $M u \in L^{p}(d \sigma)$ so that

$$
P\left[I_{k} v\right](z)=\int_{0}^{1}\left(\log \frac{1}{t}\right)^{k-1} u(t z) d t .
$$

Now it is an elementary exercise in integration by parts to show that (0.1) holds if

$$
u(z)=\left(r \frac{\partial}{\partial r}+I d\right)^{k} P\left[I_{k} v\right](r z)=(R+\bar{R}+I d)^{k} P\left[I_{k} v\right](z),
$$

where $\bar{R}=\sum_{j=1}^{n} \bar{z}_{j} \partial / \partial \bar{z}_{j}$. In other words we want to show that if $F=P\left[I_{k} v\right], v \in H^{p}, 0<p \leq 1$, then $(R+\bar{R}+I d)^{k} F$ has its admissible maximal function in $L^{p}(d \sigma)$. This is the content of this paper.

The main problem we face is that even though $F$ is a Poisson-Szegö integral its derivatives may not be. However, the results of D. Geller give us a way around this difficulty. In [8], Geller introduces a family of differential operators

$$
\Delta_{\alpha \beta}=\left(1-|z|^{2}\right)\left\{\sum_{i, j}\left(\delta_{i j}-z_{i} \bar{z}_{j}\right) \frac{\partial^{2}}{\partial z_{i} \partial \bar{z}_{j}}+\alpha R+\beta \bar{R}-\alpha \beta\right\}
$$


and a family of kernels

$$
P_{\alpha \beta}(z, \zeta)=C_{\alpha, \beta} \frac{\left(1-|z|^{2}\right)^{n+\alpha+\beta}}{(1-\langle z, \zeta\rangle)^{n+\alpha}(1-\langle\zeta, z\rangle)^{n+\beta}}, \quad z \in B^{n}, \zeta \in S .
$$

Here $\alpha, \beta \in \mathbf{C}$ and $C_{\alpha \beta}$ is an appropriate constant. Note that $\Delta_{00}$ is the invariant Laplacian of [11], and $P=P_{00}$ is the Poisson-Szegö kernel above. It is a straightforward calculation that $\Delta_{\alpha \beta} P_{\alpha \beta} \equiv 0$ (the differentiations being with respect to $z$ ) and that $P_{\alpha \beta}$ is an approximate identity as long as $\operatorname{Re}(n+\alpha+\beta)>0$, and hence for such values of $\alpha$ and $\beta$

$$
U(z)=\int_{S} P_{\alpha \beta}(z, \zeta) u(\zeta) d \sigma(\zeta)=P_{\alpha \beta}[u](z)
$$

solves the Dirichlet problem $\Delta_{\alpha \beta} U=0, U=u$ on $S$. The relevance of all this is that if $\Delta_{00} U=0$ then certain derivatives $D U$ satisfy $\Delta_{\alpha \beta} D U=0$ for appropriate $\alpha$ and $\beta$. Returning to our original problem we have $F=P\left[I_{k} v\right], v \in H^{p}, 0<p \leq 1$. We show that $(R+\bar{R}+I d)^{k} F$ can be written in the form $\sum \bar{S}_{\alpha \beta}(R, \bar{R}) F$, where $\alpha, \beta$ are non-positive integers, $|\alpha|+|\beta| \leq k, S_{\alpha \beta}(R, \bar{R})$ has degree $|\beta|$ in $R$ and $|\alpha|$ in $\bar{R}$ and $\Delta_{\alpha \beta} S_{\alpha \beta}(R, \bar{R}) F=0$. That is we write $(R+R+I d)^{k} F$ as a sum of solutions to the equations $\Delta_{\alpha \beta} U=0$. After establishing a unicity theorem for the Dirichlet problem for certain values of $\alpha, \beta$ (a unicity theorem that is already implicit in the work of C. R. Graham [10] in the case of the Heisenberg group) we see that for each $\alpha, \beta$ we have

$$
S_{\alpha, \beta}(R, \bar{R}) F(z)=P_{\alpha \beta}\left[S_{\alpha \beta}(R, \bar{R}) F\right](z) .
$$

Now we want to get into a position to apply standard techniques from harmonic analysis; singular integrals and approximate identities. For our range of $\alpha$ and $\beta, P_{\alpha \beta}$ is a smooth approximate identity and hence if $S_{\alpha \beta}(R, \bar{R}) F$ were in $H^{p}$ it would follow that $P_{\alpha \beta}\left[S_{\alpha \beta}(R, \bar{R}) F\right]$ would have its admissible maximal function in $L^{p}$, which is what we want. So what we want to show is that if $F=$ $P\left[I_{k} v\right], v \in H^{p}$, then if $j+l \leq\left. k R^{j} \bar{R}^{l} F\right|_{s}$ lies in $H^{p}$. What we mean, of course, is that the map $\left.v \rightarrow R^{j} \bar{R}^{l} P\left[I_{k} v\right]\right|_{s}$, originally defined for smooth functions, can be realized as a standard singular integral on $S$ and hence maps $H^{p}$ to $H^{p}$. We do this by exploiting an idea of $R$. Graham [10] who showed that certain radial derivatives of $U=P[u]$, when restricted to the boundary, are actually tangential. What we show is this: if $u$ is sufficiently restricted then for each 
$\alpha, \beta,|\alpha|+|\beta| \leq k$, there is a polynomial $Q_{\alpha \beta}$ in two variables, of total degree at most $|\alpha|+|\beta|$ such that

$$
\left.S_{\alpha \beta}(R, \bar{R}) P[u]\right|_{s}=Q_{\alpha \beta}(L, \bar{L}) u
$$

on $S$. Here $L, \bar{L}$ are certain tangential derivatives on $S$. Then it remains only to show that the map $v \rightarrow Q_{\alpha \beta}(L, \bar{L}) I_{k} v$ can be realized as a standard singular integral and hence maps $H^{p}$ to $H^{p}$.

We end the introduction with a few more definitions: for $i<j$,

$$
T_{i j}=\bar{z}_{i} \frac{\partial}{\partial z_{j}}-\bar{z}_{j} \frac{\partial}{\partial z_{i}}
$$

and

$$
\bar{T}_{i j}=z_{i} \frac{\partial}{\partial \bar{z}_{j}}-z_{j} \frac{\partial}{\partial \bar{z}_{i}}
$$

Then we define

$$
L=\sum_{i<j} \bar{T}_{i j} T_{i j}
$$

and

$$
\bar{L}=\sum_{i<j} T_{i j} \bar{T}_{i j}
$$

and

$$
\mathscr{L}_{0}=-\frac{1}{2}(L+\bar{L})
$$

In [8], Geller gives the following "radial-tangential" form for $\Delta_{\alpha \beta}$ :

$$
\begin{aligned}
\Delta_{\alpha \beta}=\left(1-|z|^{2}\right)\left\{\frac{1}{|z|^{2}}\left(\left(1-|z|^{2}\right) R \bar{R}-\mathscr{L}_{0}+\frac{n-1}{2}(R+\bar{R})\right)\right. \\
+\alpha R+\beta \bar{R}-\alpha \beta\} .
\end{aligned}
$$

For the definition of admissible limit we need the admissible approach region

$$
D_{\alpha}(\zeta)=\left\{z \in B^{n}:|1-\langle z, \zeta\rangle|<\frac{\alpha}{2}\left(1-|z|^{2}\right)\right\} .
$$

$f$ has an admissible limit at $\zeta$ if

$$
\lim _{z \rightarrow \zeta, z \in D_{\alpha}(\zeta)} f(z)
$$

exists for all $\alpha>0$ and the admissible maximal function $M_{\alpha} f(\zeta)$ is defined as

$$
\sup _{z \in D_{\alpha}(\zeta)}|f(z)|
$$

For the definition of non-isotropic Hausdorff measure, see [4]. 
LEMMA 1.1. If $\Delta_{\alpha \beta} f=0$ then

(i) $\Delta_{\alpha, \beta-1}(R f-\beta f)=0$,

(ii) $\Delta_{\alpha-1, \beta}(\bar{R} f-\alpha f)=0$.

Proof. That something like this should hold is suggested by (1.3) of [8]. In fact a proof can be based on formulas (1.3) and (1.12) of [8]. If this line of reasoning is followed we see that, for example,

$$
\Delta_{\alpha, \beta-1}\left(R f-\beta f+\frac{\partial f}{\partial z_{1}}\right)=0
$$

and then we need to check directly that

$$
\Delta_{\alpha, \beta-1}\left(\frac{\partial f}{\partial z_{1}}\right)=0 \text {. }
$$

It seems just as easy to check the lemma directly. This is a straightforward calculation.

Corollary. Suppose $\Delta_{00} U=0$ in $B^{n}$ and $j, l$ are non-negative integers. Then there are polynomials $F_{\alpha, \beta}(x, y)$, with degree $-\alpha$ in $x$ and $-\beta$ in $y$ such that

$$
R^{j} \bar{R}^{l} U=\sum_{|\alpha|+|\beta| \leq j+l} F_{\alpha \beta}(R, \bar{R}) U
$$

and

$$
\Delta_{\alpha \beta} F_{\alpha \beta}(R, \bar{R}) U=0
$$

in $B^{n}$.

Proof. The proof follows by induction on $j+l$, using the lemma.

In [8], Geller introduces the kernels $P_{\alpha \beta}$ which solve the Dirichlet problem for the operator $\Delta_{\alpha \beta}$. We will need to know that, at least for certain values of $\alpha, \beta$, this solution is unique. This uniqueness is implicit in the work of Graham [10]. However, since there is no proof in print we will provide one here. To that end we need the following lemma which gives the relation between the operators $\Delta_{\alpha, \beta}$ and certain automorphisms of the ball. The automorphisms are the $\varphi_{a}$ given on page 25 of [11]. $\varphi_{a}(0)=a, \varphi_{a}(a)=0, \varphi_{a}^{-1}=\varphi_{a}$, among other properties. Given $a \in B$ and $\alpha, \beta$ define

$$
h_{a}^{\alpha, \beta}(z)=(1-\langle a, z\rangle)^{\alpha}(1-\langle z, a\rangle)^{\beta} .
$$


LEMMA 1.2 .

$$
\Delta_{\alpha, \beta}\left[h_{a}^{\alpha, \beta}\left(U \circ \varphi_{a}\right)\right]=h_{a}^{\alpha, \beta}\left[\left(\Delta_{\alpha \beta} u\right) \circ \varphi_{a}\right] .
$$

(Just to be very clear, on neither side of the equation is $h_{a}^{\alpha, \beta}$ composed with $\varphi_{a}$.)

Proof. First we need the following: fix $0<r<1$ and let

$$
\varphi(z)=\left(\frac{z_{1}-r}{1-r z_{1}}, \frac{s z_{2}}{1-r z_{1}}, \ldots, \frac{s z_{n}}{1-r z_{1}}\right)
$$

where $s=\sqrt{1-r^{2}}$. Let $h(z)=\left(1-r \bar{z}_{1}\right)^{\alpha}\left(1-r z_{1}\right)^{\beta}$. We need to know that

$$
\Delta_{\alpha \beta}(h \cdot u \circ \varphi)=h \cdot\left(\Delta_{\alpha \beta} u\right) \circ \varphi .
$$

This can be done by appealing to formula (1.12) of [8] and using the dilation invariance of analogous operators $\Delta_{\alpha \beta}^{H}$ defined in the Siegel upper half space. Or it can be proved by a rather lengthy direct calculation which we omit. We will now assume (1.1) holds. Let $U$ be defined by $U(z)=-z$, and apply (1.1) to $u \circ U$ and we have the conclusion of the lemma for $a=(r, 0, \ldots, 0)$, if we take into account the fact that $\Delta_{\alpha, \beta}$ commutes with any unitary matrix. Now if we use the formula $U \varphi_{a}=\varphi_{U a} U$, which is easily verified for any unitary $U$, we have the result of the lemma.

In [8], it is shown that if $\Delta_{\alpha \beta} f=0$ in $B^{n}$ then for every $0<r<1$, we have

$$
F\left(-\alpha,-\beta ; n ; r^{2}\right) f(0)=\int_{S} f(r \zeta) d \sigma(\zeta) .
$$

Here $F(a, b ; c ; x)$ denotes the usual hypergeometric function. Now supposing that $\Delta_{\alpha \beta} u=0$, and $w \in B^{n}$ we may apply (1.2) to $u=$ $h_{w}^{\alpha \beta}\left(u \circ \varphi_{w}\right)$ to obtain

$$
g_{\alpha \beta}(r) u(w)=\int_{S} h_{w}^{\alpha \beta}(r \zeta) u\left(\varphi_{w}(r \zeta)\right) d \sigma(\zeta)
$$

where we let $g_{\alpha \beta}(r)=F\left(-\alpha,-\beta ; n ; r^{2}\right)$. We will use (1.3) to draw some conclusions about boundary behaviour and uniqueness of solutions of $\Delta_{\alpha \beta} u=0$.

LEMMA 1.3. Fix $\alpha, \beta \in \mathbf{C}$.

(i) There is a bounded $u, u \neq \equiv$ such that $\Delta_{\alpha \beta} u \equiv 0$ if and only if $g_{\alpha \beta}$ is bounded. 
(ii) There is a function $u$ continuous on $\bar{B}^{n}, u \not \equiv 0$, such that $\Delta_{\alpha \beta} u \equiv 0$ in $B^{n}$, if and only if $\lim _{r \rightarrow 1} g_{\alpha \beta}(r)$ exists.

(iii) There is a function $u$ continuous on $\bar{B}^{n}, u \equiv 0$ on $\partial \bar{B}^{n}$, $u \not \equiv 0$, and $\Delta_{\alpha \beta} u \equiv 0$ in $B^{n}$ if and only if $\lim _{r \rightarrow 1} g_{\alpha \beta}(r)$ exists and is zero.

Proof. The proof follows immediately from (1.3) and the fact that if we define $G(z)=g(|z|)$ then $\Delta_{\alpha \beta} G \equiv 0$ in $B^{n}$, a fact which is clear from the discussion on page 369 of [8]. Part (iii) tells us that if $\lim _{r \rightarrow 1} g_{\alpha, \beta}(r)$ exists and is not zero then we have uniqueness for the Dirichlet problem for $\Delta_{\alpha \beta}$, i.e. if $u_{1}, u_{2} \in C\left(\bar{B}^{n}\right)$ and $\Delta_{\alpha \beta} u_{1} \equiv$ $\Delta_{\alpha \beta} u_{2} \equiv 0$ in $B^{n}$ and $u_{1} \equiv u_{2}$ on $\partial B^{n}$ then $u_{1} \equiv u_{2}$ in $B^{n}$. Note that this is the case when $\alpha, \beta$ are real and $n+\alpha+\beta>0$.

Now assuming that $\alpha, \beta$ are non-positive integers and $n+\alpha+$ $\beta>0$, then the Dirichlet problem $\Delta_{\alpha \beta} u=0, u=f$ on $\partial B^{n}$ has a unique solution $u$, for any continuous $f$, given by $u(z)=$ $\int P_{\alpha, \beta}(z, \zeta) f(\zeta) d \sigma(\zeta)$. We want to see what this solution looks like when $f \in H(p, q)$, the space of harmonic homogeneous polynomials of bidegree $(p, q)$. As in [6], we look for a solution of the form $u(r \zeta)=h\left(r^{2}\right) f(r \zeta)$. We conclude that the function $h$ is a solution of the hypergeometric equation

$$
\begin{aligned}
t(1-t) h^{\prime \prime}(t)+[(p+q+n)-(p-\alpha+q-\beta+1) t] h^{\prime}(t) \\
-(p-\alpha)(q-\beta) h(t)=0 .
\end{aligned}
$$

The only solutions of this equation which are smooth at 0 are multiples of the hypergeometric function $F(p-\alpha, q-\beta ; p+q+n ; t)$. It follows that

$$
u(r \zeta)=\frac{F\left(p-\alpha, q-\beta ; p+q+n ; r^{2}\right)}{F(p-\alpha, q-\beta ; p+q+n ; 1)} f(\zeta) .
$$

From known properties of the hypergeometric series we have that

$$
h(r)=f_{1}(r)+f_{2}(r)(1-r)^{n+\alpha+\beta} \log (1-r)
$$

where $f_{1}, f_{2}$ are analytic at $r=1$.

Our next result shows if $\Delta_{\alpha \beta} u=0$ then, with appropriate restrictions on $\alpha$ and $\beta$, certain radial derivatives of $u$ are actually tangential. This type of phenomenon was first studied by R. Graham, [10]. 
Lemma 1.4. Suppose $\alpha, \beta \leq 0$ and $n+\alpha+\beta \geq 2$. Take $u \in$ $H(p, q)$ for some $p, q$ and let $U=P_{\alpha \beta}[u]$, then we have

$$
\begin{aligned}
\left.R U\right|_{s} & =\frac{1}{\alpha+\beta+n-1}\left\{\frac{\beta}{n-1} \bar{L}-\left(\frac{\beta+n-1}{n-1}\right) L-\alpha \beta\right\} u \\
& =q_{\alpha \beta}(L, \bar{L}) u, \\
\left.\bar{R} U\right|_{s} & =\frac{1}{\alpha+\beta+n-1}\left\{\frac{\alpha}{n-1} L-\left(\frac{\alpha+n-1}{n-1}\right) \bar{L}-\alpha \beta\right\} u \\
& =q_{\alpha \beta}(L, \bar{L}) u .
\end{aligned}
$$

Proof. Using the "radial tangential" form for $\Delta_{\alpha \beta}$ we see that

$$
\begin{aligned}
\frac{1}{|z|^{2}}\left\{\left(1-|z|^{2}\right) R \bar{R} U-\mathscr{L}_{0} U+\frac{n-1}{2}(\right. & R+\bar{R}) U\} \\
& +\alpha R U+\beta \bar{R} U-\alpha \beta U \equiv 0 .
\end{aligned}
$$

Since $n+\alpha+\beta \geq 2$, it follows from (1.4) that $R \bar{R} U=O\left(\log \frac{1}{1-r}\right)$, as $r \rightarrow 1$, and hence that $\left(1-|z|^{2}\right) R \bar{R} U \rightarrow 0$ as $|z| \rightarrow 1$. Letting $|z| \rightarrow 1$ we have, since $\mathscr{L}_{0}=-\frac{1}{2}(L+\bar{L})$,

$$
\frac{1}{2}(L+\bar{L}) U+\frac{n-1}{2}(R+\bar{R}) U+\alpha R U+\beta \bar{R} U-\alpha \beta U \equiv 0
$$

on $S$, or,

$$
\left(\frac{n-1}{2}+\alpha\right) R U+\left(\frac{n-1}{2}+\beta\right) \bar{R} U=\alpha \beta U-\frac{1}{2}(L+\bar{L}) U
$$

on $S$. But we also have that

$$
R-\bar{R}=\frac{1}{n-1}(\bar{L}-L)
$$

as differential operators. If we solve these two equations for $R U$ and $\bar{R} U$ on $S$ we get the lemma.

Corollary. Suppose $u \in H(p, q)$ for some $p, q$ and $j+l<n$. Then there is a polynomial $Q$ in 2 variables of total degree $\leq j+l$ so that if $U=P_{00}[u]$ then $\left.R^{j} \bar{R}^{l} U\right|_{s}=Q(L, \bar{L}) u$.

Proof. We do induction on $j+l$. From the corollary to Lemma 1.1 we have

$$
R^{j-1} \bar{R}^{l} U=\sum_{|\alpha|+|\beta| \leq j+l-1} F_{\alpha \beta}(R, \bar{R}) U,
$$


where $\Delta_{\alpha \beta} F_{\alpha \beta}(R, \bar{R}) U=0$. Hence $R^{j} \bar{R}^{l} U=\sum R F_{\alpha \beta}(R, \bar{R}) U$. Вy Lemma 1.4

$$
\left.R\left(F_{\alpha \beta}(R, R) U\right)\right|_{s}=\left.l(L, \bar{L})\left(F_{\alpha \beta}(R, \bar{R}) U\right)\right|_{s}
$$

where $l$ is first degree. By induction $\left.F_{\alpha \beta}(R, \bar{R}) U\right|_{s}$ is a polynomial in $L, \bar{L}$ of degree at most $j+l-1$ acting on $u$.

If we now combine the corollaries to Lemmas $1.1,1.4$ we get the following.

Theorem 1. Suppose $j+l<n$. Then there are polynomials $Q_{\alpha \beta}$, $|\alpha|+|\beta| \leq j+l$ such that if $u \in H(p, q)$ and $U=P_{00}[u]$ we have

$$
R^{j} \bar{R}^{l} U=\sum_{|\alpha|+|\beta| \leq j+l} P_{\alpha \beta}\left[Q_{\alpha \beta}(L, \bar{L}) u\right] .
$$

Proof. From the corollary to Lemma 1.1 we have

$$
R^{j} \bar{R}^{l} U=\sum_{|\alpha|+|\beta| \leq j+l} F_{\alpha \beta}(R, \bar{R}) U
$$

where $\Delta_{\alpha \beta} F_{\alpha \beta}(R, \bar{R}) U=0$. Since $j+l<n, F_{\alpha \beta}(R, \bar{R}) U \in C^{1}\left(\bar{B}^{n}\right)$ and hence by uniqueness for the Dirichlet problem we have

$$
F_{\alpha \beta}(R, \bar{R}) U=P_{\alpha \beta}\left[F_{\alpha \beta}(R, \bar{R}) U\right] .
$$

Now on $S, F_{\alpha \beta}(R, \bar{R}) U=Q_{\alpha \beta}(L, \bar{L}) u$ by the corollary to Lemma 1.4 .

We have proved the theorem for $u \in H(p, q)$, we will need to extend it to the case $u=I_{k} v$ where $v \in L^{2}$, provided $j+l \leq k$. We need to know how $I_{k}$ acts on $H(p, q)$.

LEMMA 1.5. For $v \in H(p, q)$,

$$
I_{k} v=\frac{\Gamma(k)}{\Gamma\left(\frac{n-k}{2}\right)^{2}}\left\{\left(p+\frac{n-k}{2}\right)_{k}\left(q+\frac{n-k}{2}\right)_{k}\right\}^{-1} v
$$

Proof. It is easy to check that $I_{k}(v \circ U)=\left(I_{k} v\right) \circ U$ for any unitary $U$. Since $H(p, q)$ is minimal under the action of the unitary group, it is enough to prove the lemma in case $v(\zeta)=\zeta_{1}^{p} \bar{\zeta}_{2}^{q}$. We write

$$
|1-\langle z, \zeta\rangle|^{k-n}=(1-\langle z, \zeta\rangle)^{-\left(\frac{n-k}{2}\right)}(1-\langle\zeta, z\rangle)^{-\left(\frac{n-k}{2}\right)} .
$$


If we expand each factor in a binomial series and integrate term by term we arrive at

$$
\left(I_{k} v\right)(z)=\frac{1}{\Gamma\left(\frac{n-k}{2}\right)}\left(\sum_{j=0}^{\infty} \frac{\Gamma\left(\frac{n-k}{2}+p+j\right) \Gamma\left(\frac{n-k}{2}+q+j\right)}{\Gamma(p+q+n+j) j !}\right) z_{1}^{p} \bar{z}_{2}^{q} .
$$

Recognizing the series as essentially

$$
F\left(\frac{n-k}{2}+p, \frac{n-k}{2}+q ; p+q+n ; 1\right)
$$

we arrive at the desired formula.

On the other hand if $u \in H(p, q)$ then $L u=-p(q+n-1) u$ and $\bar{L} u=-q(p+n-1) u$, see [3]. Hence if $v \in H(p, q)$ and $\operatorname{deg} Q_{\alpha \beta}(L, \bar{L}) \leq j+l=k$ then

$$
Q_{\alpha \beta}(L, \bar{L}) I_{k} v=C(p, q) v
$$

where

$$
|C(p, q)| \leq \frac{C[2 p q+(p+q)(n-1)]^{k}}{\left(p+\frac{n-k}{2}\right)_{k}\left(q+\frac{n-k}{2}\right)_{k}} \leq C
$$

independent of $p, q$.

Hence the mapping

$$
v \rightarrow Q_{\alpha \beta}(L, \bar{L}) I_{k} v
$$

extends to be a bounded map from $L^{2}$ to $L^{2}$. Moreover, when $v \in L^{2}$ then the differential operator $Q_{\alpha \beta}(L, \bar{L})$ applied to $I_{k} v$ in the sense of distributions is the same as the operator just described above. From this it follows that if $I_{k} v$ is $C^{\infty}$ on some open set $\Omega \subseteq S$ then the $Q_{\alpha \beta}(L, \bar{L}) I_{k} v$ just described and the function obtained by applying the differential operator $Q_{\alpha \beta}(L, \bar{L})$ to $I_{k} v$ agree on $\Omega$. This will be used later.

We now summarize our results so far.

THEOREM 2. Fix $k<n$, then there are polynomials $Q_{\alpha \beta}$ in 2 variables of total degree at most $k$ so that for $v \in L^{2}$ we have

$$
(R+\bar{R}+I)^{k} P_{00}\left[I_{k} v\right]=\sum_{|\alpha|+|\beta| \leq k} P_{\alpha \beta}\left[Q_{\alpha \beta}(L, \bar{L}) I_{k} v\right] .
$$

Proof. We just note that $(R+\bar{R}+I)^{k}$ is a sum of terms of the form $R^{j} \bar{R}^{l}$ with $j+l \leq k$. We just add and group like terms.

Next we want to show that the operators $Q_{\alpha \beta}(L, \bar{L}) I_{k}$, which extend to be bounded in $L^{2}$ actually extend to be bounded in $H^{p}$, $0<p \leq 1$. 
THeOREM 3. Suppose $r+s \leq k$ and let $K$ be the operator defined by $K v=L^{r} \bar{L}^{s} I_{k} v$, then $K$ is bounded in $H^{p}, 0<p \leq 1$.

Proof. We consider the smooth approximations $K_{r}$ where $I_{k}(z, \zeta)$ is replaced by $|1-r\langle z, \zeta\rangle|^{k-n}$. $K_{r}$ is a multiplier on each $H(p, q)$ and in fact if $K u=C(p, q) u$ for $u \in H(p, q)$, then

$$
K_{r} u=r^{p+q} C(p, q) u
$$

and so it follows that $\left\|K_{r} u-K u\right\|_{L^{2}} \rightarrow 0$ as $r \rightarrow 1$. If we can show that for every $(p, \infty)$ atom $a$ we have $\left\|K_{r} a\right\|_{H^{p}} \leq C$ where $C$ is independent of $r$ and $a$, then the theorem will follow in a standard way. To establish this we note the following: we calculate that for $a, b>0$ we have that

$$
\bar{T}_{i j} T_{i j}(1-r\langle\zeta, w\rangle)^{-a}(1-r\langle w, \zeta\rangle)^{-b}
$$

is a sum of two terms, one of the form

$$
\left|\zeta_{i} w_{j}-w_{j} \zeta_{i}\right|^{2}(1-r\langle\zeta, w\rangle)^{-a-1}(1-r\langle w, \zeta\rangle)^{-b-1}
$$

and the other of the form

$$
\left(\zeta_{i} \bar{w}_{i}+\zeta_{j} \bar{w}_{j}\right)(1-r\langle\zeta, w\rangle)^{-a-1}(1-r\langle w, \zeta\rangle)^{-b} .
$$

If we add on $i<j$ and use the fact that $\sum_{i<j}\left|\zeta_{i} w_{j}-\zeta_{j} w_{i}\right|^{2}=$ $1-|\langle\zeta, w\rangle|^{2}$ we see that $L_{\zeta}(1-r\langle\zeta, w\rangle)^{-a}(1-r\langle w, \zeta\rangle)^{-b}$ is a sum of terms of the form

$$
\left(1-|\langle\zeta, w\rangle|^{2}\right)(1-r\langle\zeta, w\rangle)^{-a-1}(1-r\langle w, \zeta\rangle)^{-b-1}
$$

and

$$
\langle\zeta, w\rangle(1-r\langle\zeta, w\rangle)^{-a-1}(1-r\langle w, \zeta\rangle)^{-b} .
$$

There is a similar expression for $\bar{L}_{\zeta}$. So if we apply $L_{\zeta}-\bar{L}_{\zeta}$ to

$$
(1-r\langle\zeta, w\rangle)^{\frac{k-n}{2}}(1-r\langle w, \zeta\rangle)^{\frac{k-n}{2}}
$$

we get a sum of terms of the form

$$
\left(1-|\langle\zeta, w\rangle|^{2}\right)^{l}(1-r\langle\zeta, w\rangle)^{-a}(1-r\langle w, \zeta\rangle)^{b}
$$

where $a+b-l \leq n-k+r+s$. Now if we let $D_{w}$ denote any $w$ derivative which has $k T_{i j}$ 's and $(R-\bar{R}) l$-times we see that we have

$$
\left|D_{w} K_{r}(\zeta, w)\right| \leq \frac{C}{|1-\langle\zeta, w\rangle|^{n+\frac{k}{2}+l}} .
$$

From this estimate it follows in a standard way that $K_{r}$ is uniformly bounded on $(p, \infty)$ atoms (see [4]). 
Finally we point out that in our case, $(\alpha, \beta \leq 0, n+\alpha+\beta>0) P_{\alpha \beta}$ is a smooth approximate identity and hence we have

TheOREM. For $0<p \leq 1$ we have

$$
\left\|M P_{\alpha \beta} f\right\|_{L^{p}} \leq C\|f\|_{H^{p}}
$$

Putting all these results together, as indicated in the introduction we have our main theorem.

Theorem 4. Suppose $0<k<n, k$ is a positive integer and $0<$ $p \leq 1$, and $n-k p>0$. Then there is a constant $C$ such that if $\nu$ is a measure on $S$ that satisfies $\nu(B(\zeta, \delta)) \leq \delta^{n-k p}$, then

$$
\int M_{\alpha} P\left[I_{k} \nu\right]^{p} d \nu \leq C\|v\|_{H^{p}}^{p}
$$

for all $v \in H^{p}$, all $\alpha>0$.

Corollary. For each $v \in H^{p}, 0<p \leq 1$, there is a set $E \subseteq S$ with non-isotropic Hausdorff measure zero in dimension $n-k p$ such that $F=P\left[I_{k} v\right]$ has admissible limits on $S \backslash E$.

Proof. The corollary follows from the theorem and results of $\mathrm{W}$. Cohn [5].

\section{REFERENCES}

[1] D. Adams, The classification problem associated with the Besov and TriebelLizorkin spaces, preprint.

[2] P. Ahern, Exceptional sets for holomorphic Sobolev functions, Michigan Math. J., 35 (1988), 29-41.

[3] P. Ahern and J. Bruna, Maximal and area integral characterizations of HardySobolev spaces in the unit ball of $\mathbf{C}^{n}$, Revista Mat. Iberoamericana, 4, no. 1, (1988), 123-153.

[4] P. Ahern and W. Cohn, Exceptional sets for Hardy Sobolev functions, $p>1$, Indiana University Math. J., 38, no. 2 (1989), 417-453.

[5] W. Cohn, Nonisotropic Hausdorff measure and exceptional sets for holomorphic Sobolev functions, Illinois J. Math., (to appear).

[6] G. Folland, The spherical harmonic expansion of the Poisson-Szegö kernel for the ball, Proc. Amer. Math. Soc., 47 (1975), 401-408.

[7] J. Garnett and R. Latter, The atomic decomposition for Hardy spaces in several complex variables, Duke Math. J., 45 (1978), 814-845.

[8] D. Geller, Some results on $H^{p}$ theory for the Heisenberg group, Duke Math. J., 47, no. 2 (1980), 365-390. 
[9] D. Geller, The Laplacian and the Kohn Laplacian for the sphere, J. Differential Geom., 15 (1980), 417-435.

[10] C. R. Graham, The Dirichlet problem for the Bergman Laplacian I, Comm. P.D.E., 8 (1983), 433-476.

[11] W. Rudin, Function Theory in the Unit Ball in $\mathbf{C}^{n}$, New York, Springer, 1980.

Received September 4, 1990 and in revised form March 8, 1991. The first author was partially supported by a grant from NSF. The second author was partially supported by a fellowship from Ministerio de Educación y Ciencia, Spain, and a grant from D.G.I.C.Y.T. PB89-0311.

UNIVERSITY OF WISCONSIN-MADISON

480 LINCOLN DRIVE

Madison, WI 53706

AND

UNIVERSITAT DE BARCELONA

GRAN VIA 585

08071 Barcelona, SpaIN 



\section{PACIFIC JOURNAL OF MATHEMATICS EDITORS}

V. S. VARADARAJAN

(Managing Editor)

University of California

Los Angeles, CA 90024-1555-05

Herbert Clemens

University of Utah

Salt Lake City, UT 84112

F. Michael Christ

University of California

Los Angeles, CA 90024-1555

THOMAS ENRIGHT

University of California, San Diego

La Jolla, CA 92093

\section{C. MOORE}

University of California

Berkeley, CA 94720

MARTIN SCHARLEMANN

University of California

Santa Barbara, CA 93106

HAROLD STARK

University of California, San Diego

La Jolla, CA 92093

STEVEN KERCKHOFF

Stanford University

Stanford, CA 94305

ASSOCIATE EDITORS

R. ARENS

E. F. BECKENBACH (1906-1982)

B. H. NeumanN

F. WOLF

(1904-1989)

K. YosHIDA

SUPPORTING INSTITUTIONS

UNIVERSITY OF ARIZONA

UNIVERSITY OF OREGON

UNIVERSITY OF BRITISH COLUMBIA

UNIVERSITY OF SOUTHERN CALIFORNIA

CALIFORNIA INSTITUTE OF TECHNOLOGY

UNIVERSITY OF CALIFORNIA

MONTANA STATE UNIVERSITY

STANFORD UNIVERSITY

UNIVERSITY OF NEVADA, RENO

UNIVERSITY OF HAWAII

NEW MEXICO STATE UNIVERSITY

OREGON STATE UNIVERSITY

UNIVERSITY OF UTAH

WASHINGTON STATE UNIVERSITY

UNIVERSITY OF WASHINGTON 


\section{Pacific Journal of Mathematics}

\section{Vol. 153, No. $1 \quad$ March, 1992}

Patrick Robert Ahern and Carmen Cascante, Exceptional sets for Poisson integrals of potentials on the unit sphere in $\mathbf{C}^{n}, p \leq 1 \ldots \ldots \ldots \ldots \ldots$

David Peter Blecher, The standard dual of an operator space ........... 15

Patrick Gilmer, Real algebraic curves and link cobordism .............. 31

Simon M. Goberstein, On orthodox semigroups determined by their

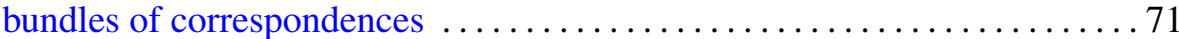

John Kalliongis and Darryl John McCullough, Homeotopy groups of irreducible 3-manifolds which may contain two-sided projective planes .......................................... 85

Yuji Konishi, Masaru Nagisa and Yasuo Watatani, Some remarks on actions of compact matrix quantum groups on $C^{*}$-algebras $\ldots \ldots \ldots \ldots 119$

Guojun Liao and Luen-Fai Tam, On the heat equation for harmonic maps from noncompact manifolds ........................... 129

John Marafino, Boundary behavior of a conformal mapping . .......... 147

Ji Min, A remark on the symmetry of solutions to nonlinear elliptic

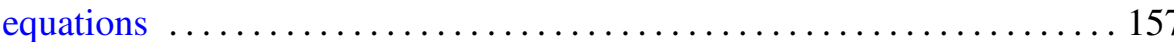

Paul Nevai and Walter Van Assche, Compact perturbations of orthogonal

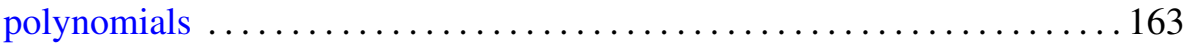

Kyril Tintarev, Level set maxima and quasilinear elliptic problems ....... 185 